\title{
Sebum component lipids penetrate through the epidermis and accumulate in the dermis
}

\author{
Marianna Lovászi ${ }^{1}$, Attila Gácsi², Lajos Kemény³ ${ }^{3}$ Erzsébet Csányi², Dániel Törőcsik ${ }^{1}$
}

\begin{abstract}
${ }^{1}$ Department of Dermatology, Faculty of Medicine, University of Debrecen, Debrecen, Hungary
${ }^{2}$ Institute of Pharmaceutical Technology and Regulatory Affairs, Faculty of Pharmacy, University of Szeged, Szeged, Hungary

${ }^{3}$ Department of Dermatology and Allergology, University of Szeged, Szeged, Hungary
\end{abstract}

INTRODUCTION: The primary function of the human sebaceous glands is to produce and secrete sebum to coat the surface of skin, aiding keratinocytes in the formation of the lipid barrier. Recent findings put forward that sebum component lipids might also have a significant contribution to the overall dermal lipid content where they could exert a (patho)physiological role.

AIMS: To determine if major sebum component lipids such as squalene, oleic-, palmitic- linoleic and stearic acid can penetrate and accumulate in the dermis when applied topically.

Figure 1

A

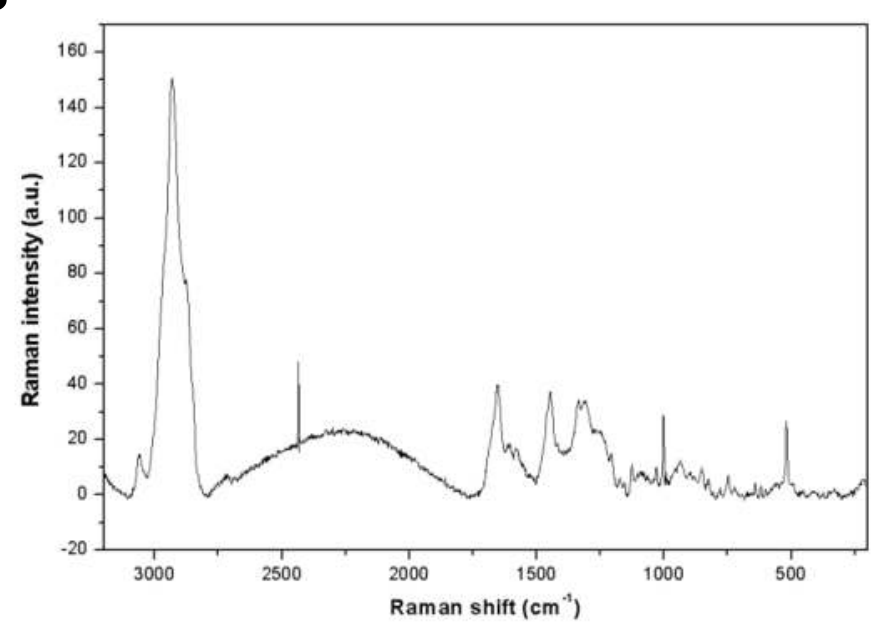

B

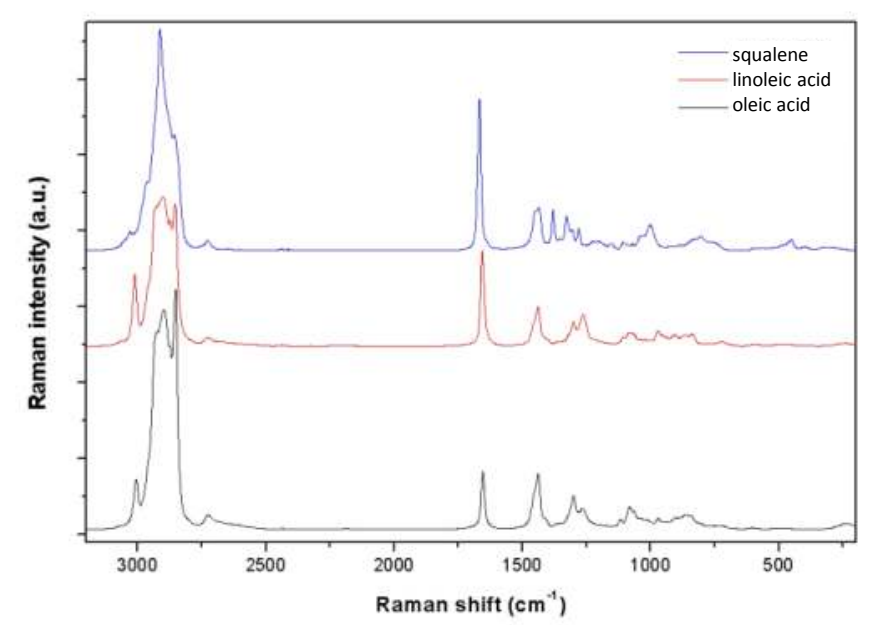

C

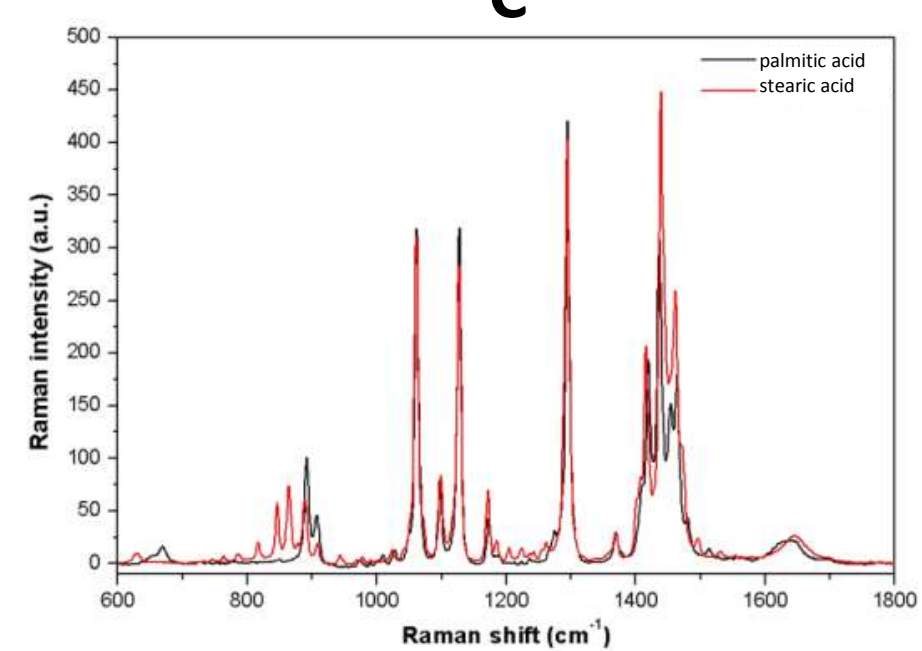

RESULTS:

First we examined the whole spectrum of an untreated human skin sample. Figure $1 \mathrm{~A}$ depicts peaks that are typical of organic samples. Since these appear in a low intensity, treatment with specific lipids that yield unique peaks are easily differentiable from native samples. Next we analyzed the Raman spectra of each lipids individually. While oleic acid, linoleic acid and squalene showed discrete patterns in accordance to the number of $C=C$ bonds (1,2 and 6 respectively), palmitic and stearic acid had nearly identical spectra (Figure 1 B, C). Therefore simultaneous quantification of the lipids was not possible however each pattern could be identified if the lipids were applied individually.

Applying squalene, oleic-, palmitic- linoleic and stearic acid individually onto skin grafts from sebaceous gland poor regions we used Raman spectroscopy to determine the extent of penetration and accumulation for each of the lipids.

Our results clearly showed that all of the tested lipids could penetrate through the epidermis without disrupting its structure (Figure 2). While palmitic acid (A) could be detected in high concentrations in the full depth of the dermis, squalene (B) and linoleic (C) acid accumulated in the upper region. Interestingly stearic acid (D) displayed a prominent enrichment in the intermediate dermal region, while oleic acid (E) was only present in moderate amounts however it showed a scattered distribution throughout the skin. From blue to red colouring the maps indicate the increasing presence of the various fatty acids in a semi-quantitative manner. Dotted lines mark the estimated dermoepidermal border.

Figure 2

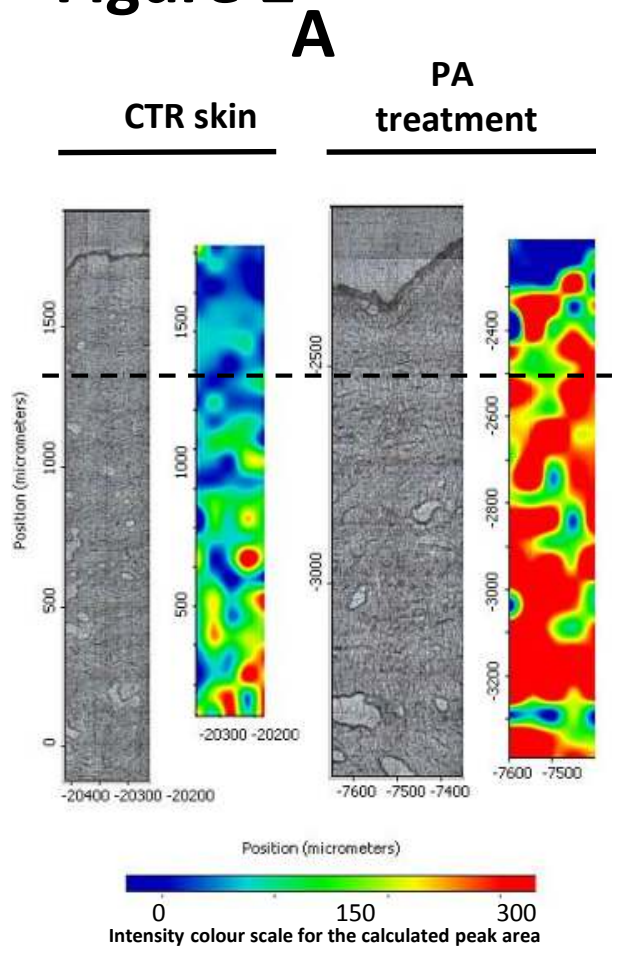

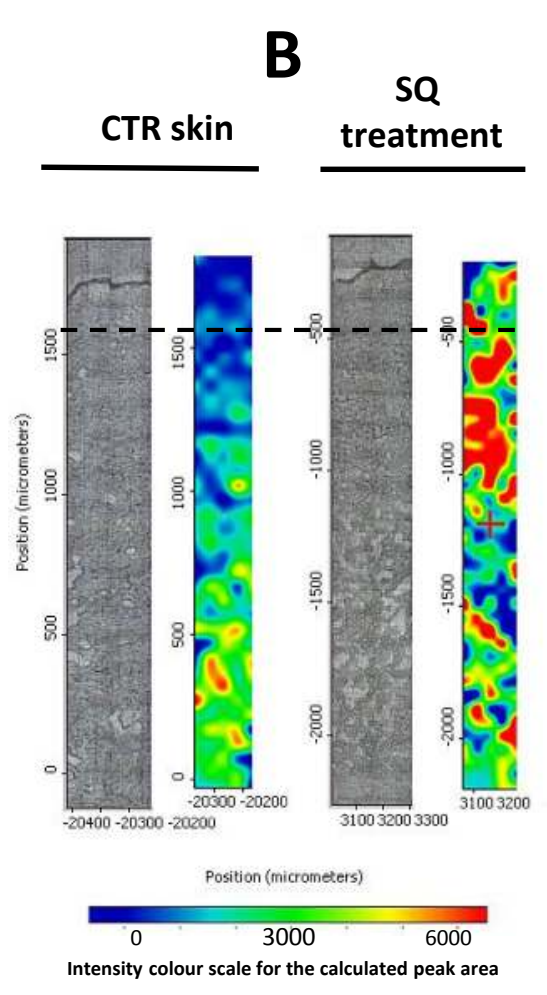

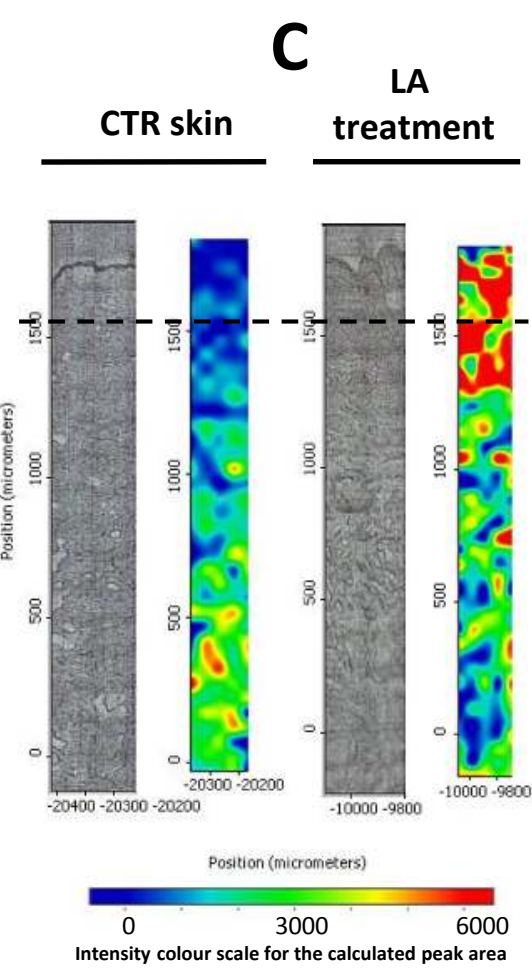

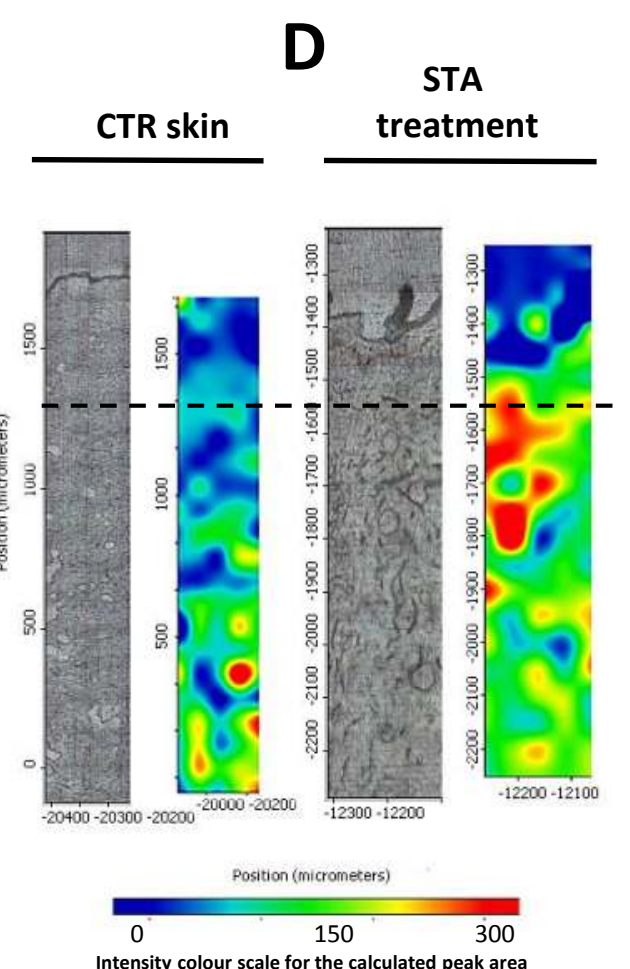

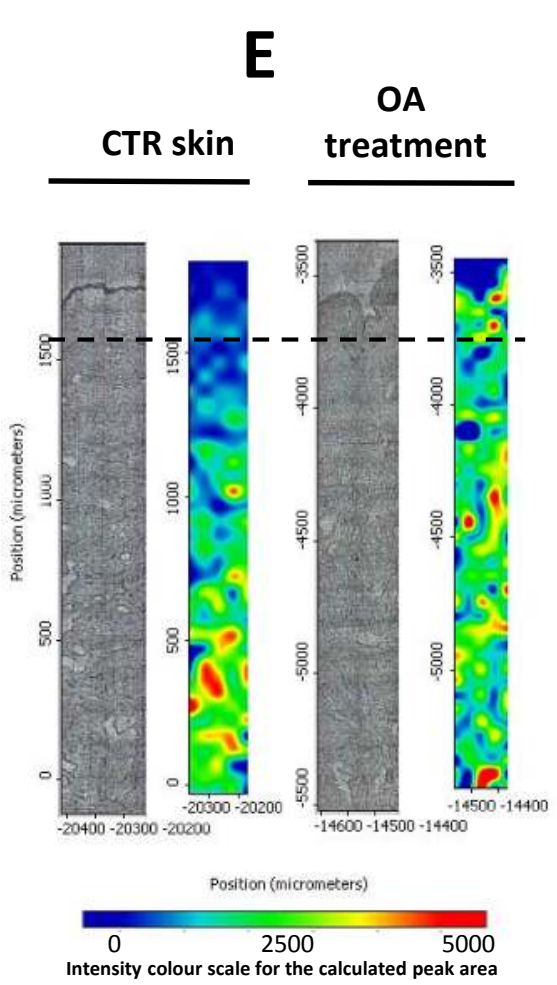

DISCUSSION: Our finding, besides extending our knowledge in considering sebum lipids more than just topical lubricants, also opens several new pathways for researchers on how these lipids could be used in therapeutics both as carriers as well as agents with direct effects.

\section{MATERIALS AND METHODS:}

\section{Raman spectroscopy}

Skin biopsies were obtained from abdominal reconstruction surgery. Approximately $1 \mathrm{~cm}^{2}$ skin samples were treated with either squalene, linoleic-, oleic-, palmitic- or stearic acid on $4 \mathrm{~mL}$ volume Franz diffusion cells using phosphate buffer saline solution to avoid skin drying. In all cases the treatment duration was $24 \mathrm{~h}$ and the treated area was 66.5 $\mathrm{mm}^{2}$. Tissue samples of the treated areas were frozen and sectioned onto aluminium coated slides. Raman spectra of each section were obtained with a DXR Raman microscope (Thermo Fisher Scientific, Waltham, MA, USA) equipped with a $532 \mathrm{~nm}$ diode laser. Instrument operation and the evaluation of measurements were done by the OMNIC Dispersive Raman 8.2 software (Thermo Fisher Scientific). In all mapping measurements 24 spectra were collected, the spectral resolution was approximately $2 \mathrm{~cm}^{-1}$, and the spectral window ranged from 200 to $3200 \mathrm{~cm}^{-1}$. The individual spectra of each fatty acid were used as a reference when comparing the treated vs untreated samples.

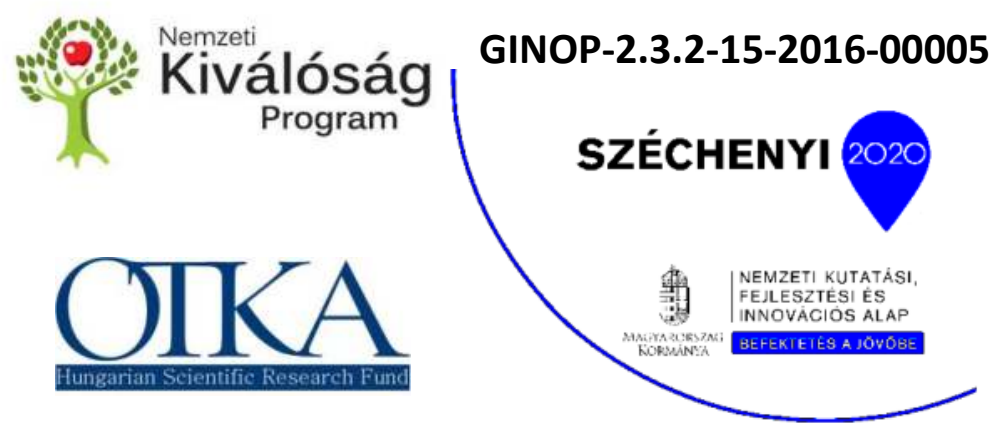

